\title{
Axion-photon conversion in neutron star magnetospheres: The role of the plasma in the Goldreich-Julian model
}

\author{
Samuel J. Witte $\odot,{ }^{1, *}$ Dion Noordhuis $\odot,{ }^{1}$ Thomas D. P. Edwards, ${ }^{2}$ and Christoph Weniger ${ }^{1, \dagger}$ \\ ${ }^{1}$ GRAPPA Institute, Institute for Theoretical Physics Amsterdam and \\ Delta Institute for Theoretical Physics, University of Amsterdam, \\ Science Park 904, 1098 XH Amsterdam, Netherlands \\ ${ }^{2}$ The Oskar Klein Centre for Cosmoparticle Physics, AlbaNova University Center, \\ Roslagstullsbacken 21, SE-10691 Stockholm, Sweden
}

(Received 30 April 2021; accepted 22 October 2021; published 24 November 2021)

\begin{abstract}
The most promising indirect search for the existence of axion dark matter uses radio telescopes to look for narrow spectral lines generated from the resonant conversion of axions in the magnetospheres of neutron stars. Unfortunately, a large list of theoretical uncertainties has prevented this search strategy from being fully accepted as robust. In this work we attempt to address major outstanding questions related to the role and impact of the plasma, including: (i) does refraction and reflection of radio photons in the magnetosphere induce strong inhomogeneities in the flux, (ii) can refraction induce premature axionphoton dephasing, (iii) to what extent do photon-plasma interactions induce a broadening of the spectral line, (iv) does the flux have a strong time dependence, and $(v)$ can radio photons sourced by axions be absorbed by the plasma. We present an end-to-end analysis pipeline based on ray-tracing that exploits a state-of-the-art auto-differentiation algorithm to propagate photons from the conversion surface to asymptotically large distances. Adopting a charge symmetric Goldreich-Julian model for the magnetosphere, we show that for reasonable parameters one should expect a strong anisotropy of the signal, refraction induced axion-photon dephasing, significant line-broadening, a variable time-dependence of the flux, and, for large enough magnetic fields, anisotropic absorption. Our simulation code is flexible enough to serve as the basis for follow-up studies with a large range of magnetosphere models.
\end{abstract}

DOI: 10.1103/PhysRevD.104.103030

\section{INTRODUCTION}

The QCD axion has emerged in recent years as one of the most compelling candidates to explain dark matter; this is predominantly because the axion is a fundamental ingredient in the most favored solution to the strong $C P$ problem [1-4], which attempts to explain why $C P$ appears to be very accurately conserved in QCD. Recent experimental advancements have allowed laboratory searches to probe vast swathes of the axion parameter space [5-8], however their progress is severely constrained by our current ignorance of the axion mass. ${ }^{1}$ Since the relic axion abundance is inherently linked to the axion mass, one

\footnotetext{
*s.j.witte@uva.nl

c.weniger@uva.nl

${ }^{1}$ This is because many searches exploit resonance effects which require tuning experimental setups to specific axion masses [9-19].

Published by the American Physical Society under the terms of the Creative Commons Attribution 4.0 International license. Further distribution of this work must maintain attribution to the author(s) and the published article's title, journal citation, and DOI. Funded by SCOAP ${ }^{3}$.
}

might hope to infer the mass directly from measurements of the cold dark matter energy density; unfortunately, difficulties in modeling topological defects self-consistently from formation to decay [20-29] (and an ignorance of the unknown scale at which the PQ symmetry was broken) lead to a broad range of estimates roughly spanning the range $10^{-6} \lesssim m_{a} \lesssim 10^{-3} \mathrm{eV}[30-32]$.

One promising search avenue which may shed light on the axion mass, and thus dramatically simplify terrestrial axion experiments, is to look for the decay and/or conversion of nonrelativistic axion dark matter into photons in astrophysical environments [33-49]. These processes yield nearly monochromatic spectral features, with the characteristic frequency of the line providing direct information about the axion mass. Axion-photon conversion can be efficient in the presence of an external magnetic field, and becomes resonantly enhanced when the background plasma has an oscillation frequency $\omega_{p} \sim{\sqrt{4 \pi \alpha n_{e} / m_{e}}}^{2}$ roughly equivalent to the mass of the axion [33]. Neutron

\footnotetext{
${ }^{2}$ More generally, the plasma mass in a cold dilute plasma is given by $\omega_{p}=\sqrt{\sum_{i} 4 \pi \alpha n_{i} / m_{i}}$, where $n_{i}$ and $m_{i}$ are the number density and mass of species $i$.
} 
stars have recently been proposed as prime targets to search for radio signals from axion-photon conversion due to their plasma rich magnetospheres and enormous magnetic fields [38-48,50]. Although potentially capable of probing QCD axion dark matter, such observations are currently impeded by large theoretical uncertainties which prevent ongoing searches from reliably estimating the expected signal.

The aforementioned uncertainties can be largely broken down into two categories: (i) those related to the astrophysical description and characterization of magnetospheres, and (ii) those related to the production and propagation of the radio photons generated from axionphoton conversion. The former of these concerns reflects an overarching ignorance of the complicated phenomena that occur in extremely energetic strong-field environments. There is promise, however, that these uncertainties may be largely mitigated in the near future, as upcoming observations from telescopes such as SKA [51-57] and advances in the simulations of magnetospheres push our understanding to new limits (see, e.g., [58-64] for recent developments in magnetosphere modeling). The latter uncertainty, encompassing open questions such as: how axions and photons mix in an inhomogeneous three-dimensional plasma; how photons propagate through the magnetosphere; if photonplasma interactions induce a broadening of the spectral line; and if these photons can be absorbed, is more readily addressable given the current knowledge of the community.

In this paper, we perform an end-to-end calculation of the radio signal in the Goldreich-Julian (GJ) magnetosphere model. That is to say, our formalism self-consistently follows individual photons from their point of genesis on the conversion surface to asymptotically large distances where they can be treated as free streaming radio waves. We do this using a state-of-the-art auto-differentiation raytracing algorithm, which allows us to carefully track axionphoton dephasing, photon reflections and refraction, energy exchanges with the ambient plasma, and photon absorption. In addition, we introduce a Monte Carlo (MC) sampling algorithm to efficiently draw photon trajectories from generic phase space distributions. We emphasize that this work contains a complete pipeline capable of computing the expected radio signature arising from axion-photon conversion in any magnetosphere model with arbitrary axion phase space distributions, and thus represents a major step toward ensuring the reliability of indirect axion searches.

\section{PHOTON PROPAGATION}

Previous attempts to compute the radio flux arising from axion-photon conversion in magnetospheres have all but ignored the complicated electrodynamics detailing how photons sourced near the neutron star surface propagate to the light cylinder ${ }^{3}$ [39-43]. A subset of the present authors made a first attempt in Ref. [43] to improve upon these assumptions by following the trajectories of individual photons, with the goal of discerning the time-variation and viewing angle sensitivity of radio observations [43]. This work, however, adopted the simplifying assumption of a free space dispersion relation (i.e., photon trajectories are unaffected by the plasma), implying that their technique could not track photon refraction, reflection, absorption, and energy losses/gains due to the time variability of the plasma, quantities which are fundamental for understanding radio telescope sensitivity. We begin here by discussing how to move past the aforementioned assumptions, and properly account for the effect of the plasma on the propagation of radio photons.

The linear response of a plasma to the presence of electromagnetic modes is fully characterized by the dielectric tensor $\epsilon_{i j}$. Once this quantity is known, the dispersion relations governing the behavior of electromagnetic modes in the plasma are obtained by solving Maxwell's equations in Fourier space; in practice, this amounts to solving for the roots of the determinant of $\left\|n^{2} \delta_{i j}-n_{i} n_{j}-\epsilon_{i j}\right\|$, where $n_{i}=k_{i} / \omega$ is the refractive index, and $\vec{k}$ and $\omega$ are respectively the wave number and frequency of the Fourier mode of interest. For a cold and neutral plasma, this determinant yields a 10th order polynomial, highlighting the fact that the plasma response can in general be quite complex and diverse [65]. In the large magnetization and low frequency limit (a good approximation for radio photons in neutron star magnetospheres), this can be reduced to a 6th order polynomial, allowing for the identification of three unique modes (each with $a \pm$ solution). These are often called the magnetosonic-t (sometimes also called the magnetoacoustic-t or X mode), the Alfvén, ${ }^{4}$ and Langmuir-O modes. In the infinite magnetic field limit, the first of these has the trivial dispersion relation $\omega^{2}=k^{2}-$ notice that semi-relativistic axions cannot excite this mode, and thus it is not of interest. The Alfvén mode is a subluminous (i.e., $n=k / \omega>1$ ) density perturbation that is unavoidably damped at large radii. The Langmuir-O mode on the other hand is a superluminous wave with both longitudinal and transverse components, and naturally evolves into a transverse ordinary "O" mode 5 as the wave propagates away from the neutron star [66]; this is the only mode that axions can excite, and thus it is this mode that we must track in our analysis. We derive in Appendix A of the

\footnotetext{
${ }^{3}$ The light cylinder is defined by the radial distance at which the corotating plasma would be required to move at the speed of light, i.e., $R_{\mathrm{LC}} \sim 1 / \omega_{\mathrm{NS}}$, where $\omega_{\mathrm{NS}}$ is the rotational frequency of the neutron star. Outside of this radius the magnetosphere model breaks down.

${ }^{4}$ This mode is synonymous with the magnetosonic-t waves in the limit of purely parallel propagation.

${ }^{5}$ Ordinary modes are those described by the conventional cold isotropic plasma dispersion relation, i.e., $\omega^{2}=k^{2}+\omega_{p}^{2}$.
} 
Supplemental Material [67] the generalized dispersion relation of the Langmuir-O mode for long wavelength radiation traveling through a highly magnetized plasma, and show an illustration of the phase diagram for each mode at various angles of propagation. In the limit that the plasma is nonrelativistic (we defer the study of a boosted plasma to Appendix A of the Supplemental Material [67]), the dispersion relation of the Langmuir-O mode is given by

$$
\omega^{2}=\frac{1}{2}\left(k^{2}+\omega_{p}^{2}+\sqrt{k^{4}+\omega_{p}^{4}+2 k^{2} \omega_{p}^{2}\left(1-2 \cos ^{2} \tilde{\theta}\right)}\right),
$$

where $\tilde{\theta}$ is the angle between the magnetic field and $\vec{k}$ (for reference, we point out that Eq. (1) is consistent with one of the roots of the dispersion relation obtained in Eq. (55) of [66], in the limit of a nonrelativistic plasma). Notice that because this is a superluminal mode, i.e., the phase velocity $v_{p} \geq 1$, we need not be worried about Landau damping [65].

In a three-dimensional inhomogeneous plasma, one can derive a set of equations (under a generalized WKB theory $^{6}$ ) that governs the evolution of a wave packet as it propagates through a medium. These are called the raytracing equations, and for a predefined dispersion relation $\omega(\vec{x}, \vec{k}, t)$ are given by [65]

$$
\begin{aligned}
& \frac{d \vec{x}}{d t}=\nabla_{k} \omega(\vec{x}, \vec{k}, t), \\
& \frac{d \vec{k}}{d t}=-\nabla_{x} \omega(\vec{x}, \vec{k}, t), \\
& \frac{d \omega}{d t}=\partial_{t} \omega(\vec{x}, \vec{k}, t),
\end{aligned}
$$

The first two equations allow for photon trajectories to be carefully tracked from deep in the magnetosphere to distances $d \sim R_{\mathrm{LC}}$, while the final of these equations defines the differential energy injected to (or imparted from) the plasma per unit path length. Integrating Eq. (4) along the trajectory yields the net energy change of the photon from production to detection.

At distances far from the surface of the neutron star, the strong-field assumption used in the derivation of the dielectric tensor breaks down; this is expected to occur when the cyclotron frequency of electrons is comparable to the photon frequency, i.e., $\Omega_{e} / \gamma \equiv q_{e} B /\left(m_{e} \gamma\right) \sim \omega$, where $\gamma$ is the boost factor of the electrons and positrons in the plasma rest frame (which we take to be one). At this point, two additional effects may arise. First, new propagating

\footnotetext{
${ }^{6}$ The WKB method (or approximation) is a procedure for finding approximate solutions to linear differential equations with space-time dependent coefficients (see e.g., [74]).
}

modes will appear, implying the dispersion relation given in Eq. (1) may no longer be valid. We have verified numerically, however, that at the distances where this occurs the dispersion relation is to a very high degree given by the free space solution, i.e., $\omega^{2}=k^{2}$. Additionally, the fact that the dispersion relation given in Eq. (1) is void of poles and resonances precludes the possibility of mode mixing (in which the propagating wave jumps from one dispersion relation to another) $[65,75,76]$. The second novel effect is resonant cyclotron absorption, in which the propagating wave excites an electron or ion to a higher Landau level. In general, this happens when $\omega-k_{\|} v_{\|}-$ $\Omega_{e} / \gamma=0$ (see e.g., [77-79]). For a nonrelativistic plasma the second term can be dropped, and the resonance occurs when $\omega \sim \Omega_{e}$. We emphasize here two crucial points. First, while the cyclotron resonance never has a significant impact on the total luminosity, it can significantly suppress the flux coming from the torus and lobes (note that the luminosity is unaffected only because the dominant contribution comes from the throats, where the optical depth is always small). Second, the cyclotron resonance becomes important for magnetic field strengths characteristic of magnetars, which implies (contrary to prior belief) that stronger magnetic fields are not always better suited for axion searches. We outline the computation of the optical depth $\tau$ and illustrate both of these points in Appendix B of the Supplemental Material [67].

Despite the simplistic form of Eq. (1), solving the raytracing equations is complicated by the nontrivial functional dependencies of $\omega_{p}$ and $\tilde{\theta}$, which are inherently determined by the magnetosphere model and the direction of photon propagation. In order to solve Eqs. (2)-(4), one needs to be able to efficiently and accurately determine the gradients of each of these quantities without adding crippling computational overhead. For this reason we employ the auto-differentiation package FORWARDDIFF [80], which enables fast derivative calculations with minimum loss in accuracy, and can be easily generalized to work for generic dispersion relations and magnetosphere models in future work. ${ }^{8}$

Throughout this work we adopt the GJ model of the magnetosphere [81], which is derived by finding the minimum charge density allowed by a stable self-consistent solution to Maxwell's equations in the presence of a strong rotating magnetic field. The charge density and magnetic field are given by

\footnotetext{
${ }^{7}$ As the propagating Langmuir-O mode approaches the branch cut at $\omega \sim \omega_{p}$, mode tunneling may be possible, but this is naturally a suppressed process [65].

${ }^{8}$ This package, and autodifferentiation in more general terms, works by augmenting the algebra into a space of multidimensional dual numbers - this new algebra allows one to iteratively apply the chain rule to find derivatives at any order and to arbitrary working precision. Importantly, this is different from symbolic differentiation, which is extremely computationally inefficient, and numerical differentiation, which is subject to rounding and discretization errors.
} 


$$
\begin{gathered}
n_{\mathrm{GJ}}(\vec{r})=\frac{2 \vec{\omega}_{\mathrm{NS}} \cdot \vec{B}}{e} \frac{1}{1-\omega_{\mathrm{NS}}^{2} r^{2} \sin ^{2} \theta}, \\
B_{r}=B_{s}\left(\frac{r_{\mathrm{NS}}}{r}\right)^{3}\left(\cos \theta_{m} \cos \theta+\sin \theta_{m} \sin \theta \cos \psi\right), \\
B_{\theta}=\frac{B_{s}}{2}\left(\frac{r_{\mathrm{NS}}}{r}\right)^{3}\left(\cos \theta_{m} \sin \theta-\sin \theta_{m} \cos \theta \cos \psi\right), \\
B_{\phi}=\frac{B_{s}}{2} \sin \theta_{m} \sin \psi .
\end{gathered}
$$

Here, $\vec{\omega}_{\mathrm{NS}}=\omega_{\mathrm{NS}} \hat{z}$ is the rotational frequency of the neutron star, $B_{s}$ is the magnetic field strength at the surface of the neutron star, $\theta_{m}$ is the misalignment angle between $\vec{B}$ and $\omega_{\mathrm{NS}}, \psi(t)=\phi-\omega_{\mathrm{NS}} t$, and $r_{\mathrm{NS}}$ is the radius of the neutron star. We assume throughout that $n_{e^{-}}=n_{\mathrm{GJ}}$; since electrons and positrons have the same effect, taking $n_{e^{+}}=0$ does not influence our results (so long as the net charge density is given by $n_{\mathrm{GJ}}$ ). It is worth mentioning that realistic magnetospheres may have large charge multiplicities $M$ (defined by $M \equiv n_{e} / n_{\mathrm{GJ}}$ ), relativistic streaming plasma's, and deviations from the dipole magnetic field configuration - we leave a thorough investigation of these effects to future work. It is also worth mentioning that non-active neutron stars are expected to be comprised almost entirely of electrons and ions, with a strong spatial separation between the charged populations (see, e.g., [41]). In this case, the results presented below are likely to only be meaningful for photons originating from the electron-dominated region, as the plasma mass for ions is suppressed relative to that of electrons.

In Fig. 1, we provide an illustration outlining the raytracing technique presented here. In this image, we show how the trajectories of photons sourced on the conversion surface of a neutron star can be accurately traced to asymptotic distances (note that in the true procedure, we track photons to distances $r \sim R_{\mathrm{LC}}$, which extends far beyond the distances illustrated in Fig. 1). Close inspection of the region near the conversion surface shows evidence of strong reflections and refraction for many of these randomly selected trajectories. After propagating photons to the light cylinder, one may map their sky location back to the conversion surface in order to better understand the sensitivity of the flux, at a given viewing angle, to modeling uncertainties in the magnetosphere. This mapping is illustrated in Fig. 1, where we assign a color to the conversion surface based on the sky location of the sourced photons at the light cylinder; here, red identifies the point of genesis for photons that propagate to $\theta \sim 0$ (i.e., the north pole) at $r \sim R_{\mathrm{LC}}$, while blue indicates photons that finish at $\theta \sim \pi$ (i.e., the south pole). One can see that photons sourced on the top of the torus (the azimuthal feature) or the northern lobe predominantly propagate upward, while photons sourced on the bottom of the torus or the southern lobe predominantly propagate downward. While

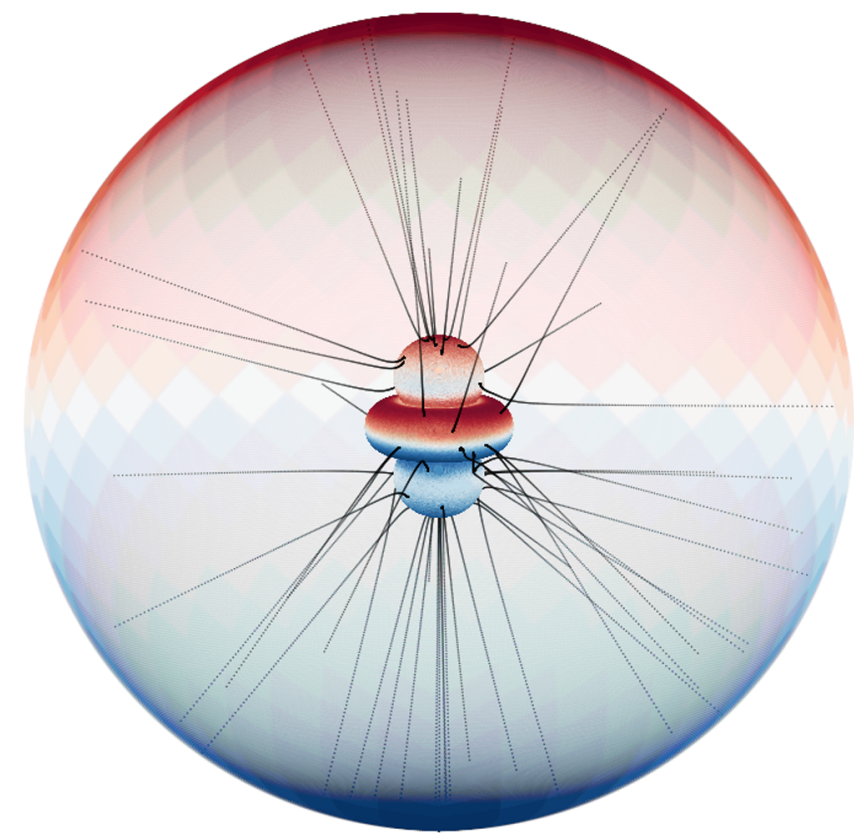

FIG. 1. Illustrative example of the ray-tracing procedure implemented in this manuscript. Photons (black lines) are sourced on the conversion surface in the magnetosphere and are propagated to distances $d=R_{\mathrm{LC}}$ (represented by large sphere). The color coding on the conversion surface denotes the sky location where photons are most likely to end up (with red/blue corresponding to north/south pole). This plot, along with all others (including an additional animation illustrating the time evolution of the ray tracing algorithm) can be reproduced with https://github.com/ SamWitte/Anteater_DataShare.

this agrees with naive expectations, a more complicated structure can be seen on the sides of the lobes and in the throats (the areas between the lobes and the torus), where axion-photon conversion can be much more efficient. We present a more systematic illustration of the photon refraction and reflection using two-dimensional projections in Appendix A of the Supplemental Material [67]. We emphasize that throughout this work we adopt a flat Minkowski metric. Using the Schwarzschild metric would induce corrections proportional to $r_{s} / r$, with $r_{s}$ being the Schwarzschild radius (note that the rotational frequency of neutron stars is never sufficiently large so as to necessitate the use of the Kerr metric [82]). At the surface of the neutron star, this amounts to a maximal correction of the metric of $\sim 10 \%$. While future high precision measurements may require the inclusion of these effects, we neglect them here for computational efficiency.

\section{PHOTON PRODUCTION RATE}

The previous section outlined the procedure for propagating photons produced from the point of conversion in the magnetosphere to the light cylinder. We now describe how to properly source these photons from an arbitrary phase space distribution. In this section we outline a novel 
approach based on MC integration which is easily generalizable to a broad range of problems in which the phase space density is nontrivial. The total rate at which photons are produced from axion-photon conversion is given by

$$
R_{a \rightarrow \gamma}=\int d^{3} v \int d \operatorname{Sn}_{a}(\vec{r}) f(\vec{r}, \vec{v})|\vec{v} \cdot \hat{n}| P_{a \rightarrow \gamma}(\vec{r}, \hat{v}),
$$

where $S$ is the conversion surface defined by the unit normal vector $\hat{n}, n_{a}$ is the axion number density, $f(\vec{r}, \vec{v})$ is the velocity phase space distribution, and $P_{a \rightarrow \gamma}$ is the axionphoton conversion probability given by $[40,43]$ (see Appendix C of the Supplemental Material [67] for a complete derivation)

$$
P_{a \rightarrow \gamma}=\frac{\pi}{2 v_{c}^{2}}\left(\frac{g_{a \gamma \gamma} B}{\sin \theta}\right)^{2}\left|\partial_{\ell} k_{\gamma}\right|^{-1} \frac{1}{\sin ^{2} \theta} .
$$

Here, $g_{\text {ary }}$ is the axion-photon coupling, $B$ the magnetic field strength, $v_{c}$ the axion velocity at the conversion surface, and $\partial_{\ell} k_{\gamma}$ the derivative of the momentum of the photon along the path length at the conversion surface. In previous work, the derivative of $k_{\gamma}$ has been truncated at leading order in velocity, yielding $\partial_{\ell} k_{\gamma} \sim \partial_{\ell} \omega_{p} /\left(v_{c} \sin ^{2} \tilde{\theta}\right)$ (see Appendix of [40]); if one further assumes radial trajectories, the derivative can be approximated by $\left|\partial_{\ell} k_{\gamma}\right| \sim 3 m_{a} /\left(2 r_{c} v_{c}\right)$, where $r_{c}$ is the radial distance of the conversion surface (see, e.g., $[40,42,43])$. Importantly, many trajectories are not radial and can have derivatives that vary significantly from this simplified assumption-in some cases by many orders of magnitude. In addition, the higher order correction in velocity carries a term proportional to $\partial_{\ell} \tilde{\theta}$, with $\tilde{\theta}$ the angle between $\vec{k}$ and the magnetic field, which may in some circumstances compensate for the velocity suppression and become the dominant term. We leave the details outlining the derivation of this expression (keeping the next-to-leading order velocity expansion intact) to Appendix C of the Supplemental Material [67]. Furthermore, we emphasize that autodifferentiation can be used to directly compute the derivatives of interest without having to resort to simplified approximation schemes.

Equation (8) has been derived under the assumption that axions and photons can be described by one-dimensional plane waves (see Appendix C of the Supplemental Material [67]). Obtaining the generalized equations describing the evolution of the mixing in three dimensions is of great interest, but we deem this to be beyond the scope of the current work. We do, however, note that the resonant conversion will be detuned if photons strongly deviate from their assumed straight line trajectories on sufficiently short timescales. Here, we attempt to account for this effect through a numerical rescaling of the conversion length as follows.

Axion-photon conversion will remain efficient while the phase overlap, defined by $\phi(z)=\int_{0}^{z} d \ell\left(k_{a}-k_{\gamma}\right)$ (where $k_{a}$ and $k_{\gamma}$ are the axion and photon momenta), remains small. For photons that travel along straight line trajectories (on scales where the conversion is active), there exists a direct mapping between the conversion length $L_{c} \equiv \sqrt{\pi /\left|\partial_{\ell} k_{\gamma}\right|}$ and the dephasing induced over that length scale-in particular, for straight line trajectories this amounts to $\phi\left(L_{c}\right) \simeq \pi / 2$. If we now take an axion trajectory oriented along the $z$-axis, the one-dimensional plane wave approximation amounts to making the substitution: $e^{i \vec{k} \cdot \vec{r}} \rightarrow e^{i k z}$. Should refraction occur, one can see that the projection of the phase onto the $z$-axis is actually given by $e^{i k z \cos \alpha}$, with $\alpha$ defining the angle between $k_{\gamma}(\ell)$ and $\hat{z}$. This is the source of the refraction induced dephasing. We approximate the impact of axion-photon dephasing by replacing $k_{\gamma} \rightarrow k_{\gamma} \cos \alpha$ in the phase $\phi$, and computing the modified conversion length $L_{c}^{\prime}$ such that $\phi\left(L_{c}^{\prime}\right)=\pi / 2$. Since $P_{a \rightarrow \gamma}$ is proportional to $L_{c}^{2}$, we then rescale the conversion probability by a factor of $\left(L_{c}^{\prime} / L_{c}\right)^{2}$. We believe this procedure represents a reasonable approximation to the impact of premature refraction induced dephasing. A more detailed description of this procedure is outlined in Appendix $\mathrm{C}$ of the Supplemental Material [67].

Equation (7) can be computed under arbitrary circumstances via a $\mathrm{MC}$ integration. We outline here the procedure of this calculation for the specific scenario where dark matter in a virialized halo falls onto the neutron star isotropically:

(1) Sample a direction $\hat{v}$ from an isotropic distribution in $\left(\theta_{v}, \phi_{v}\right)$.

(2) Define a disk $\mathcal{A}$ perpendicular to $\hat{v}$ and centered at the origin with radius $R_{\mathcal{A}} \geq R_{s, \text { max }}$, where $R_{s, \text { max }}$ is the maximum radius of the conversion surface.

(3) Randomly sample a point uniformly from the disk $\vec{r}_{\mathcal{A}}$, and define the vector passing through $\vec{r}_{\mathcal{A}}$ in the direction $\hat{v}$ as $\vec{b} .^{9}$

(4) Extend the vector $\vec{b}$ forward and backward to radii $r \geq R_{s, \max }$, and compute the plasma frequency along the vector. The points where $\omega_{p} \simeq m_{a}$ define the potential points of conversion $\vec{p}_{i}$. Notice that the aforementioned defines a generic procedure for performing an arbitrary surface integral [83]. Performing the integration over the direction of the axion velocity can be done by randomly sampling from a unit sphere, and reweighting the samples by $\hat{v} \cdot \hat{n}^{10}$

\footnotetext{
${ }^{9} \mathrm{We}$ found that convergence can be increased by using importance sampling to preferentially map out the phase space at small radii where conversion probabilities are large.

${ }^{10}$ Technically this sampling step is unnecessary, as one could just as well take $\hat{v}$ to be in the direction $\hat{b}$ (in this case the angular weight is built into the sampling itself). However, due to the fact that the conversion probability diverges in the limit $\hat{v} \cdot \hat{n} \rightarrow 0$, resampling improves convergence.
} 

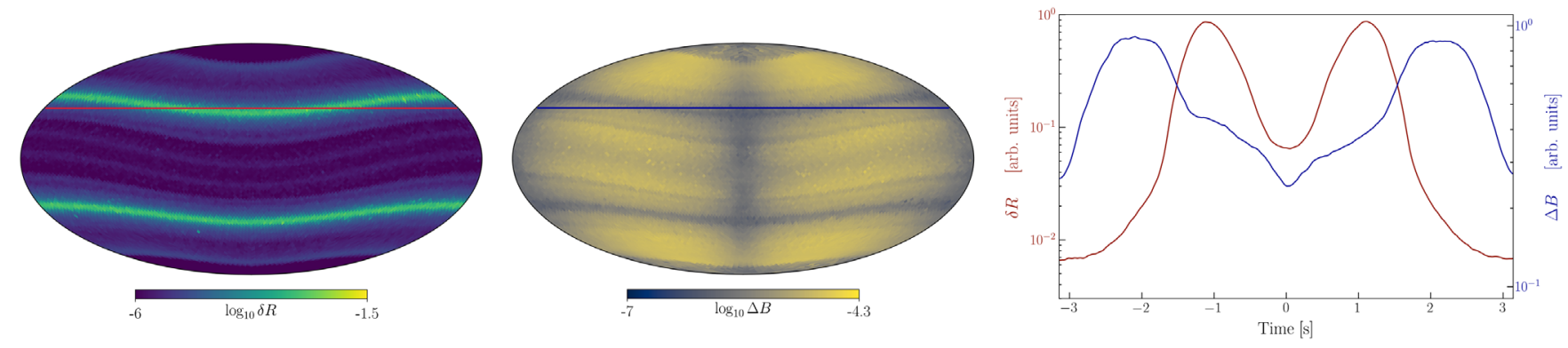

FIG. 2. Fractional rate [left, see Eq. (12)], and characteristic line width [center, see Eq. (13)] as a function of angular position in the sky after having traveled a distance $r \sim R_{\mathrm{LC}}$, with $R_{\mathrm{LC}}$ the radius of the light cylinder. Results adopt the fiducial GJ model and $m_{a}=10^{-6} \mathrm{eV}$ (see text). The time dependence of the flux (red) and line width (blue) at a fixed viewing angle of $\theta=57^{\circ}$ can be seen by tracing horizontal lines across each of the maps; this process is illustrated in the right panel.

(5) At this point only the integration over $v$ remains. Unfortunately, sampling uniformly from the local speed distribution is nontrivial. We therefore choose to use importance sampling, where we instead sample from the asymptotic speed distribution

$$
f_{\infty}\left(v_{\infty}\right)=\frac{4 \pi}{\left(\pi v_{0}^{2}\right)^{3 / 2}} v_{\infty}^{2} e^{-v_{\infty}^{2} / v_{0}^{2}}
$$

and re-weight the samples by the ratio $f(\vec{r}, \vec{v}) /$ $f_{\infty}\left(v_{\infty}\right)$.

The net result is that one can express Eq. (7) as

$R_{a \rightarrow \gamma} \simeq \frac{1}{N} \sum_{j=1}^{N} \frac{\pi R_{\mathcal{A}}^{2} \rho_{\infty}}{m_{a}} \sum_{\vec{p}_{i} \in N_{j}} \frac{\left(v_{\min }^{2}+v_{\infty}^{2}\right)}{v_{\infty}} P_{a \rightarrow \gamma}\left(\hat{v}_{\vec{p}_{i}}\right)$,

where $N$ is the number of samples drawn, $v_{\min }=$ $\sqrt{2 G M_{\mathrm{NS}} / r}, v_{\infty}$ is the sampled asymptotic speed (which we obtain using inversion sampling), and $\vec{p}_{i}$ are the points of conversion in the sample $N_{j}$. Notice that the local and asymptotic speed are related via $v^{2}=v_{\infty}^{2}+v_{\min }^{2}$. In this derivation we have adopted the local phase space distribution obtained by applying Liouville's theorem to the asymptotic velocity distribution, which can be approximately expressed as [40]

$$
f(\vec{r}, \vec{v}) \simeq \frac{1}{\left(\pi v_{0}^{2}\right)^{3 / 2}} \exp \left(\frac{2 G M_{\mathrm{NS}}}{r v_{0}^{2}}\right) \exp \left(\frac{-v^{2}}{v_{0}^{2}}\right),
$$

for $v \geq v_{\min }$. We emphasize that this procedure can be straightforwardly generalized to more complex anisotropic phase space distributions which may arise in, for example, in-falling axion miniclusters [46], binary neutron starblack hole mergers [47], and dark matter streams.

\section{APPLICATION TO GJ MODEL}

In the previous sections we defined how to generate photon seeds on the conversion surface with position and momentum $\left(\vec{x}_{i}, \vec{k}_{i}\right)$, properly weighted by the phase space density and conversion probability, and how to properly trace the position, momentum, and energy transfer of each photon as it propagates away from the neutron star. In this section we present the results of repeating this procedure over many millions of trajectories in order to generate detailed maps of the photon distributions. ${ }^{11}$ With these maps, one can understand in detail the isotropy, timedependence, viewing angle dependence, and line width of the radio signal generated from the conversion of axion dark matter around neutron stars.

We present the results of our fiducial model in this section (obtained by taking $M_{\mathrm{NS}}=1 M_{\odot}, r_{\mathrm{NS}}=10 \mathrm{~km}$, $\omega=1 \mathrm{~s}^{-1}, B_{s}=10^{14} \mathrm{G}$, and $\theta_{m}=0.2$ ), and discuss the implications of changing these fiducial values in Appendix D of the Supplemental Material [67]. Photons are all sourced at time $t=0$, and propagated to distances near the light cylinder; we have verified explicitly that increasing this threshold by orders of magnitude has no impact on the results. We then translate the position of these photons into a healpix sky map, and define the fractional rate in a pixel as

$$
\delta R_{i} \equiv \frac{R_{\text {pixel }}}{R_{\text {total }}}=\frac{\sum_{\gamma_{i} \in \text { pixel }} R_{\gamma_{i}} e^{-\tau_{\gamma_{i}}}}{\sum_{i} R_{\gamma_{i}} e^{-\tau_{\gamma_{i}}}},
$$

where $R_{\gamma_{i}}$ is given by Eq. (10), and $\tau_{\gamma_{i}}$ is the optical depth of the photon. A map of the fractional rate is illustrated in the left panel of Fig. 2. In addition to the positions of each photon, we also track the energy exchanged with the plasma during photon propagation as this may induce line broadening. Defining the final state energy of a single photon as $E_{\gamma_{i}}=\delta \omega_{\gamma_{i}}+E_{a_{i}, \infty}$, where $E_{a_{i}, \infty}$ is the asymptotic energy of the axion sourcing $\gamma_{i}$ prior to in-fall and $\delta \omega_{\gamma, i}$ being the time integration of Eq. (4), one can write the characteristic width of the spectral line in a pixel as

\footnotetext{
${ }^{11}$ We defer a discussion of the number of photons required for convergence, and the typical associated computational time, to Appendix F of the Supplemental Material [67].
} 


$$
\Delta B=\sqrt{\frac{\sum_{\gamma_{i} \in \text { pixel }} R_{\gamma_{i}} e^{-\tau_{\gamma_{i}}} \times\left(E_{\gamma_{i}}-\left\langle E_{\gamma}\right\rangle\right)^{2}}{\left\langle E_{\gamma}\right\rangle^{2} \sum_{\gamma_{i} \in \text { pixel }} R_{\gamma_{i}} e^{-\tau_{\gamma_{i}}}}} .
$$

Here $\left\langle E_{\gamma}\right\rangle$ is the typical final state photon energy, which we set to $m_{a}$ (accurate to a very high degree when averaged over the full sky). We plot this quantity in the central panel of Fig. 2. This image illustrates a number of important features. First, there is a strong anisotropy expected in the signal-depending on the position in the sky, the timeaveraged flux may vary by many orders of magnitude. Second, the time variation of the flux, which can be read off the figure by tracing horizontal lines over the period of oscillation (we illustrate this process using a red band in the left panel of Fig. 2), depends crucially on the observing angle, but can be significant in many cases. We present a more detailed analysis and discussion of the time dependence in Appendix E of the Supplemental Material [67]. Finally, the line dispersion greatly exceeds the dispersion introduced by the asymptotic axion velocity dispersion (by $\sim 1.5$ orders of magnitude in the fiducial model presented); that is to say, the line width is strongly dominated by energy exchanges with the plasma.

In general, $\Delta B$ does not uniquely characterize the line width, but rather the energy variance in the flux about the axion mass. Large values of $\Delta B$ can thus manifest either as energy shifts in the location of $\left\langle E_{\gamma}\right\rangle$ or as an actual broadening of the line about $\left\langle E_{\gamma}\right\rangle \sim m_{a}$. We have verified that for most pixels the shift in the central value of the line (away from $m_{a}$ ) is typically on the order of $33 \% \times \Delta B$, implying the dominant influence is broadening, with the shift of the line playing a small but not necessarily negligible role.

If one neglects systematic uncertainties, the flux sensitivity of a radio telescope is given by the ideal radiometer equation [84] which depends inversely on the square root of the bandwidth [84] - a large dispersion will therefore reduce the sensitivity of a line search and complicate the identification of the signal. The time dependence of both the bandwidth (blue) and flux (red) for a fixed viewing angle, taken here to be $\theta=57^{\circ}$, is shown in the right panel of Fig. 2 (using arbitrary units). While Fig. 2 is produced under fixed magnetosphere parameters, the results for the most part do not change qualitatively under variations of these parameters (although the magnitude of the time variation is sensitive to the characteristic scale of the conversion radius, which depends on the magnetosphere parameters) — perhaps the only exception being $\theta_{m}$, which can modify the isotropy of the flux. Quantitatively, however, the total luminosity, relative luminosity (i.e., the variation in the luminosity between the highest and lowest flux), and the line width may all be affected by the choice of $B_{s}, \omega_{\mathrm{NS}}, \theta_{m}$, and $m_{a}$. We defer a discussion of how variations in these parameters affect the luminosity to
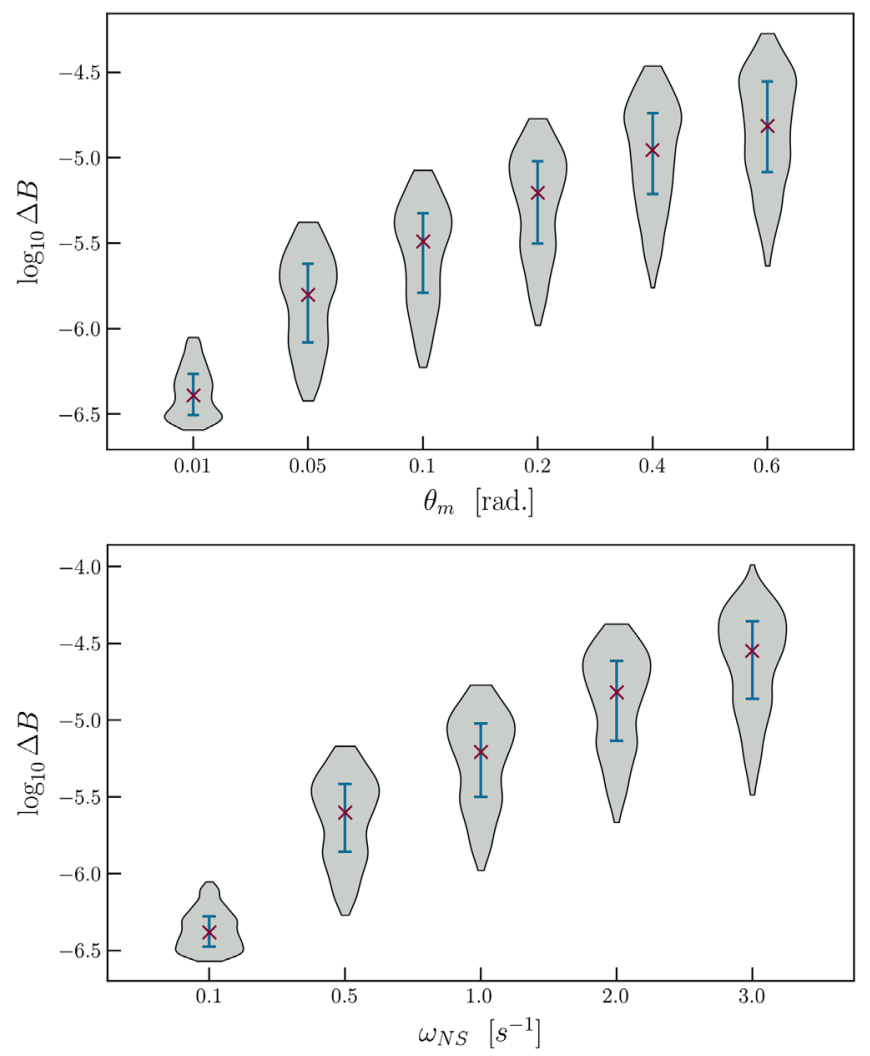

FIG. 3. Distribution of spectral line widths $\Delta B$ [see Eq. (13)] in each pixel for an $N_{\text {side }}=8$ healpix map, varying $\theta_{m}$ (top) and $\omega_{\mathrm{NS}}$ (bottom). Median value and $25 / 75 \%$ quartiles are shown in red and blue, respectively.

Appendix D of the Supplemental Material [67], and focus here only on the impact of the line width. ${ }^{12}$

In Fig. 3 we plot the pixel-to-pixel probability distribution functions of the line width $\Delta B$ as a function of $\theta_{m}$ (top) and $\omega_{\mathrm{NS}}$ (bottom), keeping the remaining parameters fixed to their fiducial values. The median and $25 / 75 \%$ quartiles are shown with red crosses and blue vertical lines, respectively. Importantly, the width also depends on the characteristic scale of the conversion surface, which may be altered by changing $m_{a}$ and $B_{s}$ (in addition to $\omega_{\mathrm{NS}}$ ). Comparing with the minimum expected line width (which can be obtained by assuming the width is given by the asymptotic axion velocity distribution, and is roughly of the order $\Delta B \sim 10^{-6}$ ), one can see that the true width across a majority of the pixels greatly exceeds previous expectations. We note here that the broadening of the line width has been previously discussed separately for reflection and refraction in Refs. [42,44] respectively. Our analysis inherently accounts for these effects, and roughly appears to agree with the linear scaling of $\Delta B$ with $\omega_{\mathrm{NS}}$ as described in [44].

\footnotetext{
${ }^{12}$ We highlight here the importance of the line width as this has been among the most debated aspects of the expected radio signal.
} 
The ultimate goal of this work is to properly assess the consequences of including non-trivial plasma phenomena for axion-based radio surveys. In order to address this point, we estimate the sensitivity of the Square Kilometer Array (SKA) to axion-photon conversion in the magnetosphere of the magnetar PSR J1745-2900, situated only $\sim 0.1 \mathrm{pc}$ from the Galactic Center [85-88]. The flux density observed by a radio telescope in a bandwidth $\Delta f$ is given by

$$
S(t, \theta, \phi)=\frac{1}{D^{2} \Delta f} \frac{d P}{d \Omega}(\theta, \phi, t),
$$

where $d P / d \Omega$ is the differential power per unit solid angle and $D$ the distance to the neutron star (taken here to be $8.5 \mathrm{kpc}$ ). In the idealized case that the noise is purely thermal, the signal-to-noise ratio (SNR) of the timeaveraged flux density $\bar{S}$ is given by the radiometer equation

$$
\mathrm{SNR}=\bar{S}(\theta, \phi) \frac{\sqrt{2 \tau_{\mathrm{obs}} \Delta f}}{\mathrm{SEFD}},
$$

where $\tau_{\text {obs }}$ is the observational time and SEFD is the system equivalent flux density (taken here to be SEFD $=0.098 \mathrm{Jy}$ [51]). Should there exist a strong time-dependence (and assuming one is able to both look for, and characterize, the strongly time-dependent signal), the SNR can be further enhanced by a factor of $\sqrt{\sigma_{\tau}^{2}}$, where $\sigma_{\tau}^{2}$ is the time-variance of the flux [43]. Notice that since $d P / d \Omega$ is proportional to the axion-photon coupling squared, the derived limit on $g_{\text {ary }}$ clearly scales as $\propto 1 / \sqrt{\mathrm{SNR}}$.

Measurements of PSR J1745-2900 have inferred a surface magnetic field strength $B_{s} \sim 1.6 \times 10^{14} \mathrm{G}$ and a rotational period $P_{\mathrm{NS}} \sim 3.76 \mathrm{~s}[85,86] .{ }^{13}$ We further assume that the dark matter density in the Galactic Center is well-described by a Navarro-Frenk-White (NFW) profile [89]; importantly, adopting a cored Burkert dark matter profile [90] (or assuming the existence of a dark matter spike [91]) may suppress (enhance) the estimated flux density by a factor of $\sim 10^{4}\left(\sim 10^{4}\right)$.

In Fig. 4, we show the projected sensitivity (defined here using a threshold of SNR $=5$ ) of SKA to axions in the mass range $m_{a} \in\left[10^{-6}, 4 \times 10^{-5}\right] \mathrm{eV},{ }^{14}$ assuming 100 hours of observing time and a bandwidth $\Delta f / f=$ $10^{-4}$ (which represents a conservative estimate based on the

\footnotetext{
${ }^{13}$ In addition, we take the mass and radius of the neutron star to be $1 M_{\odot}$ and $10 \mathrm{~km}$, respectively.

${ }^{14}$ We adopt here a lower mass threshold consistent with the minimum frequency currently used in radio searches for axions (see, e.g., [44,48,50]), and an upper threshold roughly consistent with the maximum observable frequency by SKA-Mid. For axion masses near the upper threshold, the conversion surface is very near to the surface of the neutron star itself-thus telescopes operating at higher frequencies are unlikely to offer further improvement in this direction.
}

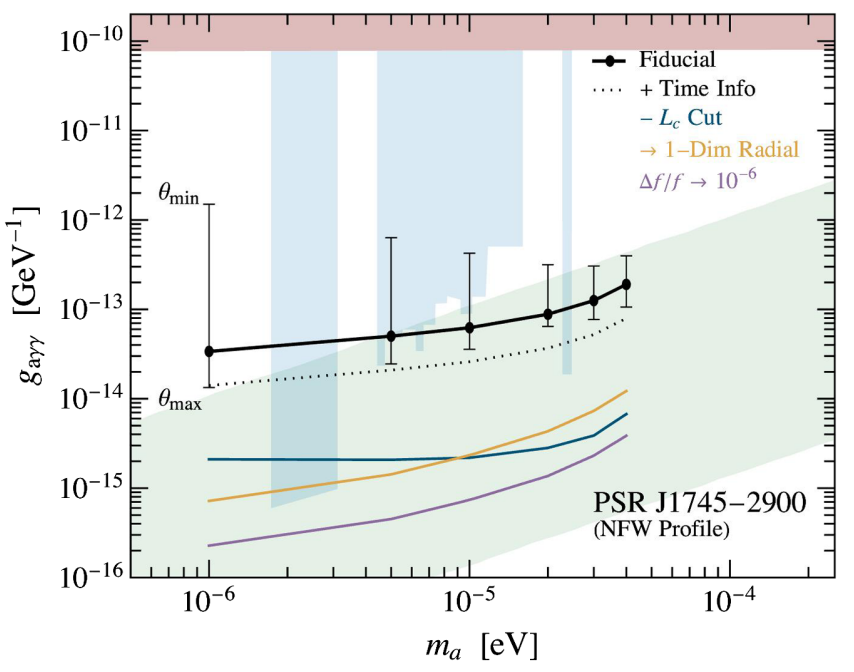

FIG. 4. Projected sensitivity to axion dark matter from SKA observations of PSR J1745-2900 (obtained requiring SNR = 5), assuming an NFW dark matter profile, 100 observing hours, and $\mathrm{SEFD}=0.098 \mathrm{Jy}$. Our fiducial sensitivity, obtained by marginalizing over the viewing angle and time-averaging, is shown with a thick black line. This can be compared with the sensitivity that one would have assuming conservatively (optimistically) the viewing angle $\theta_{\min }\left(\theta_{\max }\right)$ with the minimum (maximum) radiated power (shown using vertical error bars). We also estimate the gain in sensitivity that would occur should one assume a strongly time-varying flux (specifically we take the time variation shown in Fig. 2) - this is illustrated by the black dotted line. Our fiducial sensitivity can be compared to what would have been obtained had we: (i) not performed the conversion length cut (blue), (ii) assumed axions travel on radial trajectories and treating the conversion using the 1-dimensional formalism (in addition to the $L_{c}$ cut; labeled "1-Dim Radial," shown in yellow), and (iii) taken a bandwidth $\Delta f / f=10^{-6}$ (in addition to both of the aforementioned; purple). Existing bounds from axion haloscopes [9,10,13] (blue) and helioscopes [92] (red) are compared with the QCD axion band (green), taken from [93]. Notice that in this scenario cyclotron absorption affects the bound by no more than $10 \%$, and has been included only in the black curves.

results of Fig. 3, although we note that this choice is slightly too conservative at large masses). In our fiducial analysis we assume $\theta_{m} \sim 0.2$, a time-averaged flux density, and we marginalize over the viewing angle $\theta$. The fiducial sensitivity is shown in Fig. 4 using black dots (an interpolation is shown by the thick black line). We note that the marginalization over $\theta$ significantly biases the expected sensitivity toward lower axion-photon couplings; we illustrate this point by plotting vertical bars which represent the sensitivity from the maximally optimistic $\theta_{\max }$ and pessimistic $\theta_{\min }$ viewing angles. We also show the extent to which the sensitivity could be improved should the signal be strongly time-varying (black dotted; specifically, we assume a timevarying flux consistent with the result shown in the right panel of Fig. 2). 
In order to illustrate how each of the effects we have studied influences the expected sensitivity, we systematically remove each effect one by one, and reassess the sensitivity estimate at each step. We begin by discarding the rescaling of the conversion length, which was needed to account for the premature axion-photon dephasing induced by refraction on scales below the conversion length. The result of removing this cut is shown in Fig. 4 (blue line, labeled ' $L_{c}$ Cut'). At small masses this amounts to roughly an order of magnitude correction in the sensitivity at all masses. Next, rather than computing the conversion probability using $\partial_{\ell} k_{\gamma}$ for each individual photon, we use the simplified radial trajectory approximation adopted in previous works $\partial_{\ell} k_{\gamma} \sim 3 m_{a} /\left(2 r_{c} v_{c}\right)$, and adopt the 1-dimensional formalism in which the axion velocity is assumed to be perpendicular to the conversion surface (i.e., two of the assumptions that entered into [40]). This cut, labeled 1-Dim Radial' results in a factor of two reduction in the bound at large masses and strengthens the bound by a factor of $\sim 3$ at small masses. The strong mass dependence can at least partially be understood as a geometric effect, arising from the fact that the fraction of the conversion surface with a radially aligned normal vector increases as the conversion surface shrinks toward the surface of the neutron star (i.e., for larger axion masses). Consequently, the radial approximation becomes worse, and thus the discrepancy grows, at smaller axion masses. Finally, if the width of the radio line were set by the asymptotic axion velocity (rather than interactions with the plasma), the SNR could be enhanced by choosing a narrower bandwidth closer to $\Delta f / f \sim 10^{-6}$; making this assumption further increases the sensitivity uniformly by a factor of 3.2 (shown by the purple line). Cumulatively, our fiducial sensitivity is suppressed by a factor $\sim 10^{2}$ relative to previous estimates, and a more conservative treatment of the viewing angle may lead to a further suppression by up to one or two orders of magnitude. Finally, we note that the magnetic field of PSR J1745-2900 is not sufficiently large for cyclotron absorption to become important-the effect is never larger than a factor of two suppression in the differential power, and on average is much smaller. We include this effect in the computation of the black lines, but do not include it in the colored lines (note that it is hardly visible after marginalizing over $\theta$ ).

While the cumulative suppression in the sensitivity presented above may at first glance appear devastating for indirect axion searches, it is important to emphasize that there is still hope. In particular, we point out that the largest suppression has arisen from the uncertainty in the magnetar viewing angle. Indirect searches using neutron star populations can directly circumvent this by observing neutron stars across a wide array of possible angles (some of which are likely aligned such that the brightest flux points toward Earth). Slowly rotating and highly aligned neutron stars also experience a rather minimal amount of line broadening, implying one may still be able to use narrow bandwidths if the properties of the magnetosphere are reasonably well understood. Removing the aforementioned effects, and including the enhancement from strong time variability, one may still have sensitivity to a broad range of the axion parameter space. Importantly, we reiterate that the importance of each effect studied here is only valid in the GJ model. Further studies are required in order to understand how the conclusions found here change under reasonable assumptions of the electron and positron charge distributions.

Brief comment on Battye et al. (2021):

The preprint of Ref. [94] appeared online shortly after the publication of this work, with the goal of addressing similar questions to those discussed above. Reference [94] adopts a backward ray-tracing technique, as presented in Ref. [43], to follow photons from asymptotic distances to the conversion surface-the authors improve, however, upon Ref. [43] by (1) using the cold plasma dispersion relation $\omega^{2}=\omega_{p}^{2}+k^{2}$ (rather than the free space dispersion relation), (2) propagating photons in the Schwarzschild metric, and (3) computing line broadening effects (in a manner akin to what has been performed here). Due to the differences in methodology and treatment of the system, it is not trivial to make a direct comparison between these results. Future comparisons between the algorithm developed here and that in Ref. [94] will hopefully shed light on the importance of each effect and on the most promising approach moving forward.

\section{CONCLUSIONS}

In this work we have presented a novel tool and formalism that allows for a complete treatment of axionphoton conversion in neutron stars, tracing individual trajectories of millions of photons from their genesis to distances comparable to the light cylinder. This procedure allows for an accurate determination of the anisotropy and time-dependence of the flux as well as the width of the radio line resulting from axion-photon conversion in magnetospheres. Furthermore, our formalism is capable of accounting for axion-photon dephasing induced from photon refraction near the point of production. Our formalism is fast, highly accurate, and easily generalizable to arbitrary magnetosphere models.

The results presented here illustrate four crucial points. First, the radio flux is strongly anisotropic, implying a strong inherent sensitivity to the viewing angle. In addition, photon refraction is likely to induce a premature dephasing of the axion resonance, which will strongly suppress the flux. Next, our results indicate that the width of the line for typical neutron star parameters will be determined by the energy transfer between photons and the plasma, and is typically far greater than previously appreciated. Third, the extent to which one expects a strongly time-dependent signal crucially depends on the misalignment angle and the 
viewing angle from the neutron star. Finally, for sufficiently large magnetic field strengths, resonant cyclotron absorption may no longer be negligible, implying stronger magnetic fields do not always make ideal targets for indirect axion searches. It is now of ample importance to reevaluate existing search strategies and constraints while taking these effects into full account, which we leave to upcoming work.

We have assumed throughout this paper a simplified magnetosphere model that fails to account for plasma inhomogeneities, charge separated plasma, and relativistic streaming plasma substructure. While it is unlikely that the detailed nature of the magnetosphere will be available in the near future with sufficient resolution to capture the necessary details on the scales of interest, the methods presented here will allow for many of these uncertainties to be treated systematically in a meaningful and quantitative manner. Crucially, our results represent a complete end-toend model of the conversion process, which significantly reduces the theoretical uncertainty of the resulting flux and allows for reliable inferences of the expected radio signal.

\section{ACKNOWLEDGMENTS}

The authors would like to thank Ben Safdi, Joshua Foster, Andrea Caputo, and Edoardo Vitagliano for their useful comments and discussions on the manuscript. This work has also greatly benefited from discussions with Sebastian Baum, Matthew Lawson, M. C. David Marsh, and Alexander Millar. This work has received funding from the European Research Council (ERC) under the European Union's Horizon 2020 research and innovation programme (Grant Agreement No. 864035-Un-Dark). T. E. acknowledges support by the Vetenskapsrådet (Swedish Research Council) through Contract No. 638-2013-8993 and the Oskar Klein Centre for Cosmoparticle Physics. T. E. was also supported in part by the research environment grant 'Detecting Axion Dark Matter In The Sky And In The Lab (AxionDM)' funded by the Swedish Research Council (VR) under Grant No. Dnr 2019-02337. The computations/ data handling were/was partially enabled by resources provided by the Swedish National Infrastructure for Computing (SNIC) at HPC2N partially funded by the Swedish Research Council through Grant Agreement No. 2018-05973. We acknowledge the use of the Python scientific computing packages NUMPY $[95,96]$ and SCIPY [97], as well as the graphics environment MATPLOTLIB [98]. Finally, we acknowledge the use of the Julia autodifferentiation package ForwardDiff [80].
[1] R. D. Peccei and H. R. Quinn, $C P$ Conservation in the Presence of Instantons, Phys. Rev. Lett. 38, 1440 (1977).

[2] R. D. Peccei and H. R. Quinn, Constraints imposed by $C P$ conservation in the presence of instantons, Phys. Rev. D 16, 1791 (1977).

[3] S. Weinberg, A New Light Boson?, Phys. Rev. Lett. 40, 223 (1978).

[4] F. Wilczek, Problem of Strong $P$ and $T$ Invariance in the Presence of Instantons, Phys. Rev. Lett. 40, 279 (1978).

[5] P. Sikivie, Experimental Tests of the Invisible Axion, Phys. Rev. Lett. 51, 1415 (1983); Erratum, Phys. Rev. Lett. 52, 695 (1984).

[6] P. Sikivie, Detection rates for 'invisible' axion searches, Phys. Rev. D 32, 2988 (1985); Erratum, Phys. Rev. D 36, 974 (1987).

[7] I. G. Irastorza and J. Redondo, New experimental approaches in the search for axion-like particles, Prog. Part. Nucl. Phys. 102, 89 (2018).

[8] P. Sikivie, Invisible axion search methods, Rev. Mod. Phys. 93, 015004 (2021).

[9] C. Hagmann et al. (ADMX Collaboration), Results from a High Sensitivity Search for Cosmic Axions, Phys. Rev. Lett. 80, 2043 (1998).

[10] S. J. Asztalos et al. (ADMX Collaboration), Large scale microwave cavity search for dark matter axions, Phys. Rev. D 64, 092003 (2001).
[11] S. J. Asztalos et al. (ADMX Collaboration), A SQUIDBased Microwave Cavity Search for Dark-Matter Axions, Phys. Rev. Lett. 104, 041301 (2010).

[12] M. Silva-Feaver et al., Design overview of DM radio pathfinder experiment, IEEE Trans. Appl. Supercond. 27, 1 (2017).

[13] N. Du et al. (ADMX Collaboration), A Search for Invisible Axion Dark Matter with the Axion Dark Matter Experiment, Phys. Rev. Lett. 120, 151301 (2018).

[14] L. Zhong et al. (HAYSTAC Collaboration), Results from phase 1 of the HAYSTAC microwave cavity axion experiment, Phys. Rev. D 97, 092001 (2018).

[15] A. Caldwell, G. Dvali, B. Majorovits, A. Millar, G. Raffelt, J. Redondo, O. Reimann, F. Simon, and F. Steffen (MADMAX Working Group Collaboration), Dielectric Haloscopes: A New Way to Detect Axion Dark Matter, Phys. Rev. Lett. 118, 091801 (2017).

[16] B. Majorovits et al. (MADMAX interest Group Collaboration), MADMAX: A new road to axion dark matter detection, J. Phys. Conf. Ser. 1342, 012098 (2020).

[17] P. Brun et al. (MADMAX Collaboration), A new experimental approach to probe QCD axion dark matter in the mass range above $40 \mu \mathrm{eV}$, Eur. Phys. J. C 79, 186 (2019).

[18] J. L. Ouellet et al., First Results from ABRACADABRA$10 \mathrm{~cm}$ : A Search for Sub- $\mu \mathrm{eV}$ Axion Dark Matter, Phys. Rev. Lett. 122, 121802 (2019). 
[19] C. P. Salemi et al., The Search for Low-Mass Axion Dark Matter with ABRACADABRA-10 cm, Phys. Rev. Lett. 127, 081801 (2021).

[20] R. L. Davis, Cosmic axions from cosmic strings, Phys. Lett. B 180, 225 (1986).

[21] C. Hagmann, S. Chang, and P. Sikivie, Axion radiation from strings, Phys. Rev. D 63, 125018 (2001).

[22] O. Wantz and E. P. S. Shellard, Axion cosmology revisited, Phys. Rev. D 82, 123508 (2010).

[23] T. Hiramatsu, M. Kawasaki, K. Saikawa, and T. Sekiguchi, Production of dark matter axions from collapse of stringwall systems, Phys. Rev. D 85, 105020 (2012); Erratum, Phys. Rev. D 86, 089902 (2012).

[24] M. Kawasaki, K. Saikawa, and T. Sekiguchi, Axion dark matter from topological defects, Phys. Rev. D 91, 065014 (2015).

[25] V. B. Klaer and G. D. Moore, The dark-matter axion mass, J. Cosmol. Astropart. Phys. 11 (2017) 049.

[26] M. Gorghetto, E. Hardy, and G. Villadoro, Axions from strings: The attractive solution, J. High Energy Phys. 07 (2018) 151.

[27] M. Buschmann, J. W. Foster, and B. R. Safdi, EarlyUniverse Simulations of the Cosmological Axion, Phys. Rev. Lett. 124, 161103 (2020).

[28] M. Gorghetto, E. Hardy, and G. Villadoro, More axions from strings, SciPost Phys. 10, 050 (2021).

[29] M. Buschmann, J. W. Foster, A. Hook, A. Peterson, D. E. Willcox, W. Zhang, and B. R. Safdi, Dark matter from axion strings with adaptive mesh refinement, arXiv:2108.05368.

[30] J. Preskill, M. B. Wise, and F. Wilczek, Cosmology of the invisible axion, Phys. Lett. 120B, 127 (1983).

[31] M. Dine and W. Fischler, The not so harmless axion, Phys. Lett. 120B, 137 (1983).

[32] L. F. Abbott and P. Sikivie, A cosmological bound on the invisible axion, Phys. Lett. 120B, 133 (1983).

[33] G. Raffelt and L. Stodolsky, Mixing of the photon with low mass particles, Phys. Rev. D 37, 1237 (1988).

[34] G. Sigl, Astrophysical haloscopes, Phys. Rev. D 96, 103014 (2017).

[35] A. Caputo, C. P. n. Garay, and S. J. Witte, Looking for axion dark matter in dwarf spheroidals, Phys. Rev. D 98, 083024 (2018); 99, 089901(E) (2019).

[36] A. Caputo, M. Regis, M. Taoso, and S. J. Witte, Detecting the stimulated decay of axions at radiofrequencies, J. Cosmol. Astropart. Phys. 03 (2019) 027.

[37] O. Ghosh, J. Salvado, and J. Miralda-Escudé, Axion gegenschein: Probing back-scattering of astrophysical radio sources induced by dark matter, arXiv:2008.02729.

[38] M. S. Pshirkov and S. B. Popov, Conversion of Dark matter axions to photons in magnetospheres of neutron stars, J. Exp. Theor. Phys. 108, 384 (2009).

[39] F. P. Huang, K. Kadota, T. Sekiguchi, and H. Tashiro, Radio telescope search for the resonant conversion of cold dark matter axions from the magnetized astrophysical sources, Phys. Rev. D 97, 123001 (2018).

[40] A. Hook, Y. Kahn, B. R. Safdi, and Z. Sun, Radio Signals from Axion Dark Matter Conversion in Neutron Star Magnetospheres, Phys. Rev. Lett. 121, 241102 (2018).

[41] B. R. Safdi, Z. Sun, and A. Y. Chen, Detecting axion dark matter with radio lines from neutron star populations, Phys. Rev. D 99, 123021 (2019).
[42] R. A. Battye, B. Garbrecht, J. I. McDonald, F. Pace, and S. Srinivasan, Dark matter axion detection in the radio/mm-waveband, Phys. Rev. D 102, 023504 (2020).

[43] M. Leroy, M. Chianese, T. D. P. Edwards, and C. Weniger, Radio signal of axion-photon conversion in neutron stars: A ray tracing analysis, Phys. Rev. D 101, 123003 (2020).

[44] J. W. Foster, Y. Kahn, O. Macias, Z. Sun, R. P. Eatough, V. I. Kondratiev, W. M. Peters, C. Weniger, and B. R. Safdi, Green Bank and Effelsberg Radio Telescope Searches for Axion Dark Matter Conversion in Neutron Star Magnetospheres, Phys. Rev. Lett. 125, 171301 (2020).

[45] J. H. Buckley, P. S. B. Dev, F. Ferrer, and F. P. Huang, Fast radio bursts from axion stars moving through pulsar magnetospheres, Phys. Rev. D 103, 043015 (2021).

[46] T. D. P. Edwards, B. J. Kavanagh, L. Visinelli, and C. Weniger, Transient Radio Signatures from Neutron Star Encounters with QCD Axion Miniclusters, Phys. Rev. Lett. 127, 131103 (2021).

[47] T. D. P. Edwards, M. Chianese, B. J. Kavanagh, S. M. Nissanke, and C. Weniger, Unique Multimessenger Signal of QCD Axion Dark Matter, Phys. Rev. Lett. 124, 161101 (2020).

[48] J. Darling, New limits on axionic dark matter from the magnetar PSR J1745-2900, Astrophys. J. Lett. 900, L28 (2020).

[49] S. Nurmi, E. D. Schiappacasse, and T. T. Yanagida, Radio signatures from encounters between neutron stars and QCDaxion minihalos around primordial black holes, J. Cosmol. Astropart. Phys. 09 (2021) 004.

[50] J. Darling, Search for Axionic Dark Matter Using the Magnetar PSR J1745-2900, Phys. Rev. Lett. 125, 121103 (2020).

[51] SKA System Baseline Design, https://www.skatelescope .org/key-documents/ (2021).

[52] M. Kramer, A. G. Lyne, J. T. O’Brien, C. A. Jordan, and D. R. Lorimer, A periodically active pulsar giving insight into magnetospheric physics, Science 312, 549 (2006).

[53] S. Konar, M. Bagchi, D. Bandyopadhyay, S. Banik, D. Bhattacharya, S. Bhattacharyya, R. Gangadhara, A. Gopakumar, Y. Gupta, B. Joshi et al., Neutron star physics in the square kilometre array era: An indian perspective, J. Astrophys. Astron. 37, 36 (2016).

[54] T. M. Tauris, V. M. Kaspi, R. P. Breton, A. T. Deller, E. F. Keane, M. Kramer, D. R. Lorimer, M. A. McLaughlin, A. Possenti, P. S. Ray et al., Understanding the neutron star population with the SKA, Proc. Sci. AASKA14 (2015) 039 [arXiv:1501.00005].

[55] E. F. Keane et al., A cosmic census of radio pulsars with the SKA, Proc. Sci. AASKA14 (2015) 040 [arXiv:1501.00056].

[56] A. Karastergiou et al., Understanding pulsar magnetospheres with the SKA, Proc. Sci. AASKA14 (2015) 038 [arXiv:1501.00126].

[57] J. Antoniadis, L. Guillemot, A. Possenti, S. Bogdanov, J. D. Gelfand, M. Kramer, R. Mignani, B. Stappers, and P. Torne, Multi-wavelength, multi-messenger pulsar science in the SKA era, Proc. Sci. AASKA14 (2015) 157 [arXiv:1501.05591].

[58] A. A. Philippov, A. Spitkovsky, and B. Cerutti, Ab initio pulsar magnetosphere: Three-dimensional particle-in-cell simulations of oblique pulsars, Astrophys. J. Lett. 801, L19 (2015). 
[59] J. Pétri, Theory of pulsar magnetosphere and wind, J. Plasma Phys. 82, 635820502 (2016).

[60] B. Cerutti and A. M. Beloborodov, Electrodynamics of pulsar magnetospheres, Space Sci. Rev. 207, 111 (2017).

[61] A. Y. Chen and A. M. Beloborodov, Particle-in-cell simulations of the twisted magnetospheres of magnetars. I., Astrophys. J. 844, 133 (2017).

[62] G. Brambilla, C. Kalapotharakos, A. N. Timokhin, A. K. Harding, and D. Kazanas, Electron-positron pair flow and current composition in the pulsar magnetosphere, Astrophys. J. 858, 81 (2018).

[63] F. Carrasco and M. Shibata, Magnetosphere of an orbiting neutron star, Phys. Rev. D 101, 063017 (2020).

[64] A. Y. Chen, F. Cruz, and A. Spitkovsky, Filling the magnetospheres of weak pulsars, Astrophys. J. 889, 69 (2020).

[65] D. G. Swanson, Plasma Waves (Elsevier, New York, 2012).

[66] M. Gedalin, D. Melrose, and E. Gruman, Long waves in a relativistic pair plasma in a strong magnetic field, Phys. Rev. E 57, 3399 (1998).

[67] Please see Supplemental Material at http://link.aps.org/ supplemental/10.1103/PhysRevD.104.103030 for relevant derivations, checks, and tests, as well as a broader explanation of how the results presented in the main text depend on the underlying model of the neutron star, which includes Refs. [68-73].

[68] M. Gedalin, Nonlinear waves in hot magnetized plasma, Phys. Plasmas 1, 1159 (1994).

[69] M. Lyutikov, Waves in a one-dimensional magnetized relativistic pair plasma, Mon. Not. R. Astron. Soc. 293, 447 (1998).

[70] M. Gedalin, E. Gruman, and D. Melrose, Low-frequency waves in asymmetric magnetized relativistic pair plasma, Mon. Not. R. Astron. Soc. 325, 715 (2001).

[71] J. Arons and J. J. Barnard, Wave propagation in pulsar magnetospheres-dispersion relations and normal modes of plasmas in superstrong magnetic fields, Astrophys. J. 302, 120 (1986).

[72] S. L. Adler, J. Gamboa, F. Méndez, and J. López-Sarrión, Axions and "light shining through a wall": A detailed theoretical analysis, Ann. Phys. (Amsterdam) 323, 2851 (2008).

[73] A. J. Millar, S. Baum, M. Lawson, and M. C. D. Marsh, Axion-photon conversion in strongly magnetised plasmas, arXiv:2107.07399.

[74] C. M. Bender and S. A. Orszag, Advanced Mathematical Methods for Scientists and Engineers I: Asymptotic Methods and Perturbation Theory (Springer Science \& Business Media, New York, 2013).

[75] V. Fuchs, K. Ko, and A. Bers, Theory of mode-conversion in weakly inhomogeneous plasma, Phys. Fluids 24, 1251 (1981).

[76] D. G. Swanson, Radio frequency heating in the ioncyclotron range of frequencies, Phys. Fluids 28, 2645 (1985).

[77] R. Blandford and E. Scharlemann, On the scattering and absorption of electromagnetic radiation within pulsar magnetospheres, Mon. Not. R. Astron. Soc. 174, 59 (1976).

[78] D. Melrose, Propagation effects on the polarization of pulsar radio emission, Aust. J. Phys. 32, 61 (1979).
[79] Q. Luo and D. Melrose, Cyclotron absorption of radio emission within pulsar magnetospheres, Mon. Not. R. Astron. Soc. 325, 187 (2001).

[80] J. Revels, M. Lubin, and T. Papamarkou, Forward-mode automatic differentiation in Julia, arXiv:1607.07892.

[81] P. Goldreich and W. H. Julian, Pulsar electrodynamics, Astrophys. J. 157, 869 (1969).

[82] D. Ravenhall and C. J. Pethick, Neutron star moments of inertia, Astrophys. J. 424, 846 (1994).

[83] J. A. Detwiler, R. Henning, R. A. Johnson, and M. G. Marino, A generic surface sampler for Monte Carlo simulations, IEEE Trans. Nucl. Sci. 55, 2329 (2008).

[84] R. Dewey, G. Stokes, D. Segelstein, J. Taylor, and J. Weisberg, The period distribution of pulsars, in Birth and Evolution of Neutron Stars: Issues Raised by Millisecond Pulsars, edited by S. P. Reynolds and D. R. Stinebring (International Nuclear Information System, Vienna, 1984), p. 234.

[85] J. A. Kennea et al., Swift discovery of a new soft gamma repeater, SGR J1745-29, near Sagittarius A*, Astrophys. J. Lett. 770, L24 (2013).

[86] K. Mori et al., NuSTAR discovery of a 3.76-second transient magnetar near Sagittarius A*, Astrophys. J. Lett. 770, L23 (2013).

[87] R. M. Shannon and S. Johnston, Radio properties of the magnetar near Sagittarius A* from observations with the Australia telescope compact array, Mon. Not. R. Astron. Soc. 435, L29 (2013).

[88] R. P. Eatough et al., A strong magnetic field around the supermassive black hole at the centre of the Galaxy, Nature (London) 501, 391 (2013).

[89] J. F. Navarro, The structure of cold dark matter halos, in Symposium-International Astronomical Union (Cambridge University Press, Cambridge, England, 1996), Vol. 171, pp. 255-258.

[90] F. Nesti and P. Salucci, The dark matter halo of the Milky Way, AD 2013, J. Cosmol. Astropart. Phys. 07 (2013) 016.

[91] T. Lacroix, Dynamical constraints on a dark matter spike at the galactic centre from stellar orbits, Astron. Astrophys. 619, A46 (2018).

[92] V. Anastassopoulos et al. (CAST Collaboration), New CAST limit on the axion-photon interaction, Nat. Phys. 13, 584 (2017).

[93] L. Di Luzio, F. Mescia, and E. Nardi, Redefining the Axion Window, Phys. Rev. Lett. 118, 031801 (2017).

[94] R. A. Battye, B. Garbrecht, J. I. Mcdonald, and S. Srinivasan, Radio line properties of axion dark matter conversion in neutron stars, J. High Energy Phys. 09 (2021) 105.

[95] T. Oliphant, NumPy: A Guide to NumPy (Trelgol Publishing, USA, 2006), http://www.numpy.org/.

[96] C. R. Harris et al., Array programming with NumPy, Nature (London) 585, 357 (2020).

[97] P. Virtanen et al., SciPy 1.0: Fundamental algorithms for scientific computing in Python, Nat. Methods 17, 261 (2020).

[98] J. D. Hunter, Matplotlib: A 2d graphics environment, Comput. Sci. Eng. 9, 90 (2007). 